\title{
EFECTO DE DOS TIPOS DE FUNDAS SOBRE EL FRUTO DE BANANO (Musa AAA) ${ }^{1}$
}

\author{
Alfonso Vargas-Calvo ${ }^{2}$, Henry Valle-Ruiz ${ }^{2}$
}

\section{RESUMEN}

Efecto de dos tipos de fundas sobre el fruto de banano (Musa AAA). El objetivo de este estudio fue determinar el efecto de dos fundas en la protección del racimo de banano (Musa AAA). En dos épocas climáticas (adversa y favorable) bajo condiciones del Caribe de Costa Rica se evaluaron dos fundas: 1- azul Santa Lucía (bifentrina $0,1 \%$, polietileno de $12,7 \mu$ de grosor, con perforaciones de $4 \mathrm{~mm}$ y $86,4 \mathrm{~cm}$ de ancho) y 2- transparente con aditivos para filtrar la luz ultravioleta e infrarroja (bifentrina $0,1 \%, 20,3 \mu$ de grosor, con perforaciones de $4 \mathrm{~mm}$ y $88,9 \mathrm{~cm}$ de ancho). El peso del racimo así como el grosor y la longitud del fruto central de la fila externa en la segunda, cuarta y sexta mano no difirieron ( $P>0,0556)$ entre ambas fundas. Tampoco hubo diferencias entre estas en la apariencia del racimo $(\mathrm{P}>0,4699)$ ni en la firmeza de la cáscara en grado 1 de maduración $(\mathrm{P}=0,6268)$. En las variables de medición del color del fruto solamente $\mathrm{L}^{*}$ presentó un valor $(56,48)$ más alto $(\mathrm{P}=0,0109)$ con la funda transparente fotosensible, mientras que las otras dos variables relacionadas $\left(\mathrm{a}^{*} \mathrm{y} \mathrm{b}^{*}\right)$ no fueron diferentes $(\mathrm{P}>0,1011)$ entre las fundas. El incremento adicional de 7,6 $\mu$ en el grosor del polietileno de la funda transparente con respecto a la azul Santa Lucía, no ocasionó un incremento productivo ni una mejor apariencia del racimo de banano y sus frutos.

Palabras clave: Dimensiones del fruto, Musa AAA polietileno, presentación del fruto, subgrupo Cavendish.

\begin{abstract}
Effect of two banana (Musa AAA) bunch covers on fruit quality. The objective of this study consisted to determine the effect of two protective covers of the banana (Musa AAA) bunch. The study was carried out in two climatic seasons (adverse and favorable) at the Caribbean of Costa Rica. The covers compared were: 1-blue Santa Lucia (bifentrin $0.1 \%$, polyethylene $12.7 \mu$ thickness, with perforations of $4 \mathrm{~mm}$ and $86.4 \mathrm{~cm}$ wide) and 2- transparent photosensitive (bifentrin 0,1\%, $20.3 \mu, 4 \mathrm{~mm}$ holes and 86.4 $\mathrm{cm}$ wide). The bunch weight and the thickness and length of the central fruit of the outer row in the second, fourth and sixth hands did not differ $(\mathrm{P}>0.0556)$ between bunch covers. There were no differences between bunch covers in bunch appearance $(\mathrm{P}>0.4699)$ or in the peel firmness in grade 1 of maturation $(\mathrm{P}>0.6268)$. For fruit color parameters, only $\mathrm{L}^{*}$ showed a greater $(\mathrm{P}=0.0109)$ value $(56.48)$ in the transparent photosensitive bunch cover relative to the blue Santa Lucia, whereas the other two related variables $\left(a^{*}\right.$ and $\left.b^{*}\right)$ did not differ between treatments $(\mathrm{P}>0.1011)$. The increment of 7.6 $\mu$ in the polythene thickness of the transparent photosensitive with respect to the blue Santa Lucia bunch covers did not result in increased production or in improvement of bunch and fruit appearance.
\end{abstract}

Key words: Cavendish subgroup, fruit appearance, fruit dimensions, Musa AAA, polyethylene.

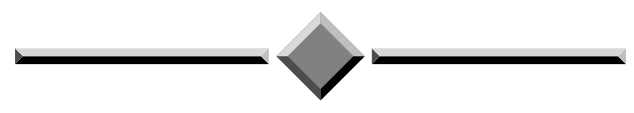

Recibido: 9 setiembre, 2010. Aceptado: 16 de mayo, 2011.

2 Corporación Bananera Nacional (CORBANA, S.A.). Limón, Costa Rica. Apdo. postal 506-2763-3055. alfvarga@corbana.co.cr; hvalle@ corbana.co.cr 


\section{INTRODUCCIÓN}

El embolse de los racimos de banano comparado con la no ejecución de la labor aumentó, especialmente en épocas de frío, el rendimiento del racimo hasta un $25 \%$, dada la diferencia de entre 2 a $6^{\circ} \mathrm{C}$ de temperatura con el medio exterior (Daniells y Lindsay 2005). Incrementos de peso entre racimos embolsados y sin embolsar también fueron encontrados por Daniells et al. (1992) y Robinson (1996) para un 9 y 16,7\% más respectivamente, en ambos casos con el $c v$ Williams. Los anteriores autores coinciden que este resultado es producto de un aumento en la longitud del fruto, el cual fue similar al de Robinson (1996) quien reportó un $10 \%$. También la práctica presentó resultados satisfactorios con relación al control de plagas y al efecto abrasivo de hojas y productos químicos (Daniells et al. 1992, Robinson 1996, Chillet y Jannoyer 1996, Ministry of Fisheries, Crops and Livestock 2003). Sin embargo, se dio la reducción del intervalo floracióncosecha de cinco a once días (Lara 1970, Daniells et al. 1987 y Daniells et al. 1992), y el aumento del largo y diámetro de los frutos y del peso del racimo (Lara 1970, Campbell y Williams 1976, Daniells et al. 1987 y 1992, Robinson 1996), fueron quizás los resultados secundarios que crearon mayor expectativa e hicieron que esta labor se expandiera en la actividad bananera (Soto 1992).

El uso de fundas cobertoras de polietileno se inició en el Caribe de Costa Rica, de acuerdo con Lara (1970) en 1963 con el propósito de controlar mecánicamente los ataques del trips de la mancha roja (Chaetanaphothrips orchidii) y se convirtió en una práctica común en la actividad bananera. En la actualidad, la labor es considerada esencial para mejorar el rendimiento (peso del racimo, dimensiones del fruto y días de la floración a la cosecha) y la apariencia del fruto de las plantaciones comerciales dedicadas a la exportación (Daniells et al. 1992, Soto 1992, Johns 1996, Robinson 1996).

El color del polietileno actúa como filtro de la Radiación Fotosintéticamente Activa (RFA) incidente, definida como aquella energía que es capaz de ser interceptada por las superficies y volúmenes de tejidos que conforman el dosel de las plantas (Guevara y Rodríguez 2006). Cayón (2007) indica que, bajo una condición de RFA incidente de $2298 \mu \mathrm{mol} / \mathrm{s} / \mathrm{m}^{2}$, el polietileno azul permite la transmisión del $73 \%$ de las longitudes de onda de la RFA hacia el interior de la funda (RFA transmitida), y el polietileno sin color (transparente), transmite el 93,5 de la RFA incidente.

Robinson (1996) evaluó en banano diferentes colores de fundas y solo encontró una respuesta productiva al color azul, dado que según dicho autor, este color permite la transmisión de calor pero reduce el daño por la quemadura de sol, mediante la limitación al máximo (Wade et al. 1993) de la radiación ultravioleta. Asimismo, Galán Sauco et al. (1996) con bananos del subgrupo Cavendish (Musa AAA, $c v$ Pequeña Enana) y bajo condiciones subtropicales de las Islas Canarias indican también que las fundas de color azul se comportaron mejor bajo condiciones de mayor altitud (racimos con más peso y mejores dimensiones del fruto así como un intervalo de cosecha más corto) que los otros colores (blanco y blancoplateado) evaluados. Sin embargo, Chillet y Jannoyer (1996) y Jannoyer y Chillet (1998) determinaron en bananos del subgrupo Cavendish (Grande Naine) con el uso de fundas de color azul, una menor longitud $(1,32$ y $0,63 \mathrm{~cm}$ menos para las manos primera y cuarta respectivamente) y peso del fruto $(25,4$ y 12,0 $\mathrm{cm}$ menos para el peso de las manos primera y cuarta, respectivamente) en comparación, con aquellos embolsados con fundas sin color (transparente) y con aditivos para reducir la luz ultravioleta e infrarroja, en ambos casos con un grosor del polietileno similar $(20$ a $22 \mu)$ y cosechados ambos a una misma edad. $\mathrm{El}$ aumento en la productividad de la funda sin color dado por un mayor peso y dimensiones de los frutos, no estuvo acompañado, de acuerdo con Chillet y Jannoyer (1996), por un deterioro de la firmeza de la cáscara, la cual fue de una magnitud similar entre ambos tipos de fundas.

En racimos embolsados con fundas de polietileno de diferente color (azul, verde y rosado) aquellos provenientes del color azul mostraron una disminución del color verde del fruto, que sin embargo, no ocasionó un efecto importante en su apariencia (Soto 1992). En contraste, los provenientes de las fundas sin color (transparente) presentaron frutos más verdes y con una cáscara más resistente.

Sin embargo, en bananos del $c v$ Mysoore (Musa $\mathrm{AAB})$ no se detectó un efecto de fundas con color (azul o blanco) y sin color (transparente), sobre el peso del racimo o de sus frutos, pero sí con respecto a la apariencia externa del racimo, en donde los 
provenientes de las fundas sin color fueron más propensos bajo condiciones tropicales a daños por quema de sol (Weerasinghe y Ruwanpathirana 2002).

El objetivo del presente estudio consistió en determinar el efecto de dos tipos de fundas en la protección del racimo de banano (Musa AAA $c v$ Williams).

\section{MATERIALES Y MÉTODOS}

Se realizaron dos experimentos en una finca comercial de banano para la exportación ubicada en el Caribe de Costa Rica (provincia de Limón, cantón de Pococí) entre diciembre del 2008 y marzo del 2009 (experimento 1 ) y entre junio y setiembre del 2009 (experimento 2), considerados respectivamente como épocas climáticas adversa y favorable para el crecimiento de la planta y el desarrollo del racimo, ambas mencionadas por Vargas y Blanco (2004) y Serrano et al. (2008). La época favorable estuvo constituida por los períodos del año lluvioso y frío (noviembre, diciembre y enero) y moderadamente seco y caliente (febrero, marzo y abril). La época adversa por su parte estuvo determinada por los períodos del año caliente y lluvioso (mayo, junio y julio) y seco y fresco (agosto, setiembre y octubre).

La finca presenta suelos de origen volcánico y fue sembrada en 1963. El área del estudio se renovó en el año 1998 con el $c v$ Williams en una densidad de 1750 plantas/ha. La precipitación total del período experimental fue $1028 \mathrm{~mm}$ (exp. 1) y $932 \mathrm{~mm}$ (exp. 2), las temperaturas promedio máxima y mínima fueron 29,0 y $17,0^{\circ} \mathrm{C}$ (exp. 1$)$ y 32,0 y $21,0^{\circ} \mathrm{C}$ (exp. 2) y la humedad relativa promedio de 88,5 (exp. 1) y $95,4 \%$ (exp. 2). El suelo, de origen volcánico presenta las siguientes características químicas: $\mathrm{pH}$ 6,04. Acidez 0,13; Ca 5,98; Mg 1,21; K 1,03 (cmol(+)/l. P 20; Fe 75; $\mathrm{Cu} 2 ; \mathrm{Zn} \mathrm{1,4;} \mathrm{Mn} 18$ (mg/l). Materia orgánica $5,34 \%$. Es considerado como Franco arcillo arenoso con porcentajes de arena, arcilla y limo de 51, 24 y 25 , respectivamente.

La fertilización se efectuó mediante la adición anual de $354 \mathrm{~kg}$ de N, $24 \mathrm{~kg}$ de $\mathrm{P}_{2} \mathrm{O}_{5}, 406 \mathrm{~kg}$ de $\mathrm{K}_{2} \mathrm{O}$, $163 \mathrm{~kg}$ de $\mathrm{MgO}, 147 \mathrm{de} \mathrm{CaO}, 78 \mathrm{~kg}$ de S, $3 \mathrm{~kg}$ de $\mathrm{B}$, $10 \mathrm{~kg}$ de $\mathrm{Zn}$ y $23 \mathrm{~kg}$ de $\mathrm{SiO}_{2} /$ ha fraccionados en 19 ciclos.

El combate de la Sigatoka negra (Mycosphaerella fijiensis) se realizó mediante la aspersión aérea de fungicidas sistémicos y protectores. Los mismos fueron aplicados rotativamente y en mezcla con aceite mineral (5-10 1/ha) de acuerdo con los requerimientos y estrategias de combate propias de cada época climática para un promedio anual de 48 ciclos de aplicación. Operaciones semanales de deshoja sanitaria se utilizaron como complemento al combate químico.

En cada experimento se estudió un grupo de inflorescencias que emergieron en una misma semana y fueron embolsadas dos semanas después junto con la eliminación de la mano de transición o mano falsa y generalmente de la inflorescencia masculina conocida como chira o bellota. No se realizó remoción de frutos laterales en cada mano del racimo pero sí la remoción manual en el campo del periantio, los estambres y el estilo terminal, que persisten adheridos al fruto del banano o desflora.

$\mathrm{Al}$ momento de embolsar los racimos con la funda de polietileno correspondiente, y para prevenir la presencia de Pseudococcus elisae se anudó al raquis, entre el espacio comprendido entre la segunda y tercera mano, una cinta de polipropileno impregnada con clorpirifos al $1 \%$ y seis semanas después se repitió la operación en la sección inferior del raquis correspondiente a la última mano cubierta por la funda.

Los tratamientos fueron los siguientes: 1- funda de color azul Santa Lucía y 2- funda transparente fotoselectiva (Cuadro 1). Los tratamientos se aplicaron cuando todas las manos del racimo estuvieron en posición horizontal (dos semanas después de la floración) en racimos de diez y nueve manos verdaderas la remoción o desmane de tres y dos de ellas, respectivamente para un tamaño de racimo en ambos casos de siete manos verdaderas. Los tratamientos se asignaron de manera alterna de acuerdo con la aparición de uno u otro tamaño de racimo. El número de racimos

Cuadro 1. Características de las fundas para cubrir el racimo de banano (Musa AAA) usadas en los experimentos. Pococí, Limón, Costa Rica. 2008-2009.

\begin{tabular}{ccccc}
\hline $\begin{array}{c}\text { Tipo de } \\
\text { funda }\end{array}$ & $\begin{array}{c}\text { Insecticida de } \\
\text { impregnación } \\
(\boldsymbol{\%})\end{array}$ & $\begin{array}{c}\text { Grosor } \\
(\boldsymbol{\mu})^{\mathbf{1}}\end{array}$ & $\begin{array}{c}\text { Perfora- } \\
\text { ción }(\mathbf{m m})\end{array}$ & $\begin{array}{c}\text { Ancho } \\
(\mathbf{c m})\end{array}$ \\
\hline Santa Lucía & bifentrina 0,1 & 12,7 & 4,0 & 86,4 \\
Fotosensible & bifentrina 0,1 & 20,3 & 4,0 & 88,9 \\
\hline
\end{tabular}

${ }^{1}$ Micras. 
embolsados por tratamiento y experimento fue de 23 en el experimento 1 y de 25 en el experimento 2 . De esta cantidad, 21 (tratamiento 1) y 19 (tratamiento 2) de ellos en el experimento $1 \mathrm{y}, 19$ (tratamiento 1) y 18 (tratamiento 2) en el experimento 2, fueron considerados. Esta cantidad correspondió con igual número de observaciones para todas las variables medidas con excepción de la RFA donde el número de observaciones fue de 16 para cada uno de los tratamientos del experimento 1 y de 12 observaciones para cada uno de los tratamientos del experimento 2 .

Las variables medidas fueron: al embolse, la Radiación Fotosintéticamente Activa (RFA) tanto incidente como transmitida $\left(\mu \mathrm{mol} / \mathrm{s} / \mathrm{m}^{2}\right)$; y a la cosecha, el peso $(\mathrm{kg})$ y la apariencia del racimo, el diámetro $(1 / 32$ ") y la longitud $(\mathrm{cm})$ del fruto central de la fila externa en la segunda, cuarta y sexta mano, la firmeza de la cáscara (lb) del fruto lateral de la fila externa de la segunda mano en grado 1 de maduración (escala de von Loesecke), y el color de la cáscara del fruto central de la fila externa de la segunda mano (esta última variable medida solamente en el experimento 2).

La cosecha se realizó en una sola etapa, a las doce semanas del embolse en ambos experimentos. El embolse se realizó una semana después de la floración.

La RFA incidente se midió sobre el dosel de la planta y la RFA transmitida se determinó dentro de la funda. Los datos de la RFA se almacenaron en un dataLogger modelo LI-1000 al cual se le adicionó un sensor Quantum LI-190SA el cual determina la densidad del flujo de fotones $\left(\mu \mathrm{mol} / \mathrm{s} / \mathrm{m}^{2}\right)$.

El peso del racimo y del pinzote se registró con la ayuda de una romana con capacidad para $50 \mathrm{~kg}$ graduada cada $200 \mathrm{~g}$. El grosor del fruto se midió en la parte media del mismo, perpendicularmente al plano de la curvatura, con la ayuda de un calibrador graduado en $1 / 32$ de pulgada ( 1 unidad $\mathrm{o}$ grado $=$ $0,794 \mathrm{~mm}$ ). La longitud se midió a lo largo de la parte externa, desde la zona de unión del pedúnculo con la pulpa, hasta el ápice (de "pulpa a punta") con una cinta métrica de plástico graduada en $\mathrm{mm}$. La medición del color de la cáscara se efectuó mediante un colorímetro portátil que determinó los valores de $\mathrm{L}^{*}, \mathrm{a}^{*}, \mathrm{~b}^{*}$ de acuerdo con la escala de CIELAB definida en 1976 por el Centro de Investigación del Color o Commission Internationale de l'Eclairage (CIE) situado en Francia, donde la coordenada $\mathrm{L}^{*}$ es una medida de la claridad (blanco-negro y varía desde el punto de ausencia de reflejo, $\mathrm{L}^{*}=0$, hasta el punto de reflejo difuso perfecto, $L^{*}=100$ ). La escala $a^{*}$ cambia desde los valores negativos para el color verde, hasta los positivos para el rojo. La escala $b^{*}$ varía desde los valores negativos para el color azul, hasta los positivos para el amarillo. La apariencia del racimo se registró mediante una escala que consideró las siguientes condiciones: 1- $\sin$ manchas o lesiones, 2- con pocas manchas o lesiones pequeñas, 3- con pocas manchas o lesiones grandes, 4- con muchas manchas o lesiones pequeñas, 5- con muchas manchas o lesiones grandes, 6- totalmente cubierto de manchas o lesiones (tejido necrosado).

La medición de la firmeza de la cáscara en grado 1 (escala de Von Loesecke 1950) de maduración (frutos de color verde) se efectuó en un fruto lateral izquierdo de la segunda mano de racimos de banano tomados al azar con la ayuda de un penetrómetro con punta cónica (libras). Los valores de firmeza obtenidos en libras fueron convertidos a Newtons mediante su multiplicación por un factor de 4,4448 .

El conjunto de datos correspondiente a RFA transmitida se analizó bajo un modelo factorial de una finca $\mathrm{x}$ dos épocas climáticas $\mathrm{x}$ dos tipos de fundas mediante el programa estadístico InfoStat (2002). Los datos de la RFA incidente se expresaron como un promedio de referencia.

Los datos de producción y firmeza de la cáscara fueron analizados mediante un ANDEVA y los de apariencia mediante una prueba de chi-cuadrado de Mantel-Haenszel mediante el programa estadístico SAS (2002-2003).

\section{RESULTADOS Y DISCUSIÓN}

En presencia de una RFA incidente de $2457 \mu \mathrm{mol} /$ $\mathrm{s} / \mathrm{m}^{2}$ en la época climática adversa y, de $2493 \mu \mathrm{mol} / \mathrm{s} /$ $\mathrm{m}^{2}$ en la época climática favorable, la funda transparente fotoselectiva permitió un mayor $(\mathrm{P}<0,0300$ y $\mathrm{P}<$ 0,0002; respectivamente) paso de luz $\left(474 \mu \mathrm{mol} / \mathrm{s} / \mathrm{m}^{2}\right.$ para un $19 \%$ de transmitancia en la época adversa y $541 \mu \mathrm{mol} / \mathrm{s} / \mathrm{m}^{2}$ para un $22 \%$ en la época favorable) con respecto a la funda de color azul denominada Santa Lucía $\left(247 \mu \mathrm{mol} / \mathrm{s} / \mathrm{m}^{2}\right.$ para un $10 \%$ de transmitancia en la época adversa y $358 \mu \mathrm{mol} / \mathrm{s} / \mathrm{m}^{2}$ para un $14 \%$ en la época favorable).

Los valores de la RFA incidente y transmitida que se determinaron en este estudio bajo las condiciones 
del Caribe de Costa Rica en el $c v$ Williams, fueron de menor magnitud que los indicados por Daza y Cayón (2006) en condiciones de Urabá (Colombia) con el $c v$ Grande Naine tanto para las fundas de color azul como sin color (transparentes). Esto podría estar asociado a una menor RFA transmitida y a una mayor densidad de plantas/ha (1750) en el área de estudio del Caribe, con respecto a la mayor RFA transmitida y menor densidad de plantas/ha (1666) señalados por Daza y Cayón (2006) en la región de Urabá. Independientemente de la magnitud, la RFA transmitida presentó un comportamiento similar a los resultados descritos por Daza y Cayón (2006) donde el polietileno de color azul permitió el paso de una menor cantidad de luz al racimo que aquel transparente. Estos últimos autores indican que la magnitud de la longitud de onda que ingresa al interior de la funda está relacionada con el color del polietileno y mencionan que la funda de color azul favorece un incremento en la temperatura interna de la funda, mientras que Chillet y Jannover (1996) observaron que las fundas transparentes fotoselectivas son más permeables a la luz roja indispensable para los procesos fisiológicos de crecimiento y desarrollo, y tienen una permeabilidad reducida a la luz infrarroja, la cual incrementa el calor que afecta dichos procesos y causa quemaduras en los frutos.

Dado que en el presente estudio la medición de la RFA transmitida se efectuó inmediatamente después del embolse, sería importante repetir dicha medición inmediatamente antes de la cosecha con el propósito de determinar si en la funda, durante el desarrollo del racimo, merced a su exposición a las condiciones ambientales, ocurre alguna degradación del color o del aditivo que pueda reducir las diferencias en la cantidad de RFA transmitida entre las fundas con color (azul) y sin color (transparente).

El peso del racimo así como el grosor y la longitud del fruto central de la segunda mano no difirieron $(\mathrm{P}>$ $0,0556)$ entre las fundas azul Santa Lucía y transparente fotoselectiva (Cuadro 2). Tampoco hubo diferencias en la apariencia del racimo ( $\mathrm{P}>0,4699$; Cuadro 3) ni en la firmeza de la cáscara en grado 1 de maduración ( $\mathrm{P}=$ 0,6268; Cuadro 4).

Para las variables de medición del color del fruto solamente $\mathrm{L}^{*}$ presentó un valor más alto con la funda transparente fotosensible $(\mathrm{P}=0,0109)$ con una tonalidad verde más brillante que la funda de color azul, mientras que las otras dos variables relacionadas $\left(\mathrm{a}^{*} \mathrm{y} \mathrm{b}^{*}\right)$ no fueron diferentes ( $P>0,1011)$ entre las fundas (Cuadro 4).

Ello indica que, a pesar de la diferencia en la longitud de onda transmitida en el color, el grosor y en los aditivos del polietileno entre la funda azul y la transparente fotoselectiva, no fue posible determinar diferencias en las variables de producción ni en la apariencia del racimo entre ambas. Este resultado difiere de aquellos encontrados por Daza y Cayón

Cuadro 2. Peso del racimo, grosor y largo del fruto central de $2^{\text {da }}, 4^{\text {ta }}$ y $6^{\text {ta }}$ manos de racimos de banano (Musa AAA) de dos experimentos, embolsados con dos tipos de fundas. El número de observaciones fue de 21 (tratamiento 1) y 19 (tratamiento 2) en el experimento 1 , y de 19 (tratamiento 1) y 18 (tratamiento 2) en el experimento 2. Pococí, Limón, Costa Rica 2008-2009

\begin{tabular}{|c|c|c|c|c|c|c|c|}
\hline \multirow{2}{*}{ Tipo de funda } & \multirow{2}{*}{$\begin{array}{l}\text { Peso del ra- } \\
\text { cimo (kg) }\end{array}$} & \multicolumn{3}{|c|}{ Grosor (1/32) } & \multicolumn{3}{|c|}{ Largo (cm) } \\
\hline & & $2^{\mathrm{da}}$ & $4^{\text {ta }}$ & $6^{a}$ & $2^{\mathrm{da}}$ & $4^{\text {ta }}$ & $6^{a}$ \\
\hline \multicolumn{8}{|l|}{ Experimento 1} \\
\hline Santa Lucía & 17,5 & 41,6 & 40,9 & 39,9 & 24,5 & 23,1 & 21,2 \\
\hline Fotosensible & 16,9 & 42,2 & 40,4 & 40,2 & 24,2 & 22,8 & 20,4 \\
\hline Error estándar & 0,79 & 0,5 & 0,5 & 0,5 & 0,4 & 0,4 & 0,3 \\
\hline Probabilidad & 0,4813 & 0,3140 & 0,4065 & 0,5844 & 0,4816 & 0,5677 & 0,0556 \\
\hline \multicolumn{8}{|l|}{ Experimento 2} \\
\hline Santa Lucía & 26,0 & 47,7 & 46,3 & 45,3 & 24,5 & 23,0 & 20,8 \\
\hline Fotosensible & 26,3 & 47,5 & 46,6 & 45,4 & 24,9 & 23,3 & 21,5 \\
\hline Error estándar & 0,5 & 0,5 & 0,4 & 0,4 & 0,4 & 0,3 & 0,3 \\
\hline Probabilidad & 0,6969 & 0,7068 & 0,6077 & 0,8147 & 0,4703 & 0,5069 & 0,1561 \\
\hline
\end{tabular}


Cuadro 3. Incidencia (\%) de lesiones de grado 1 y 2 (apariencia de frutos) en racimos de banano (Musa AAA) de dos experimentos, embolsados con dos tipos de fundas de polietileno. El número de observaciones fue de 21 (tratamiento 1) y 19 (tratamiento 2) en el experimento 1 , y de 19 (tratamiento 1 ) y 18 (tratamiento 2) en el experimento 2. Pococí, Limón,Costa Rica 20082009.

\begin{tabular}{lcccc}
\hline Tipo de funda & \multicolumn{2}{c}{ Experimento 1 } & \multicolumn{2}{c}{ Experimento 2 } \\
\cline { 2 - 5 } & Grado1 $^{\mathbf{1}}$ & Grado 2 & Grado 1 & Grado 2 \\
\hline Santa Lucía & 53 & 47 & 42 & 58 \\
Fotosensible & 63 & 37 & 44 & 56 \\
Probabilidad & \multicolumn{2}{c}{0,4966} & \multicolumn{2}{c}{0,4699} \\
\hline
\end{tabular}

${ }^{1}$ No hubo lesiones de grado mayor a 2 en ningún experimento. Grado 1 corresponde a racimos sin manchas o lesiones y grado 2 a racimos con pocas manchas o lesiones pequeñas.

Cuadro 4. Firmeza (N) y color de la cáscara del fruto central de la segunda mano según escala L*, a*, b* (grado 1 de maduración) de racimos de banano (Musa AAA) embolsados con fundas de polietileno azul Santa Lucía y transparente fotosensible. El número de observaciones para la firmeza fue de 21 (tratamiento1) y 19 (tratamiento 2) en el experimento 1 , y de 19 (tratamiento 1) y 18 (tratamiento 2 ) en el experimento 2 . Para el color fue de19 (tratamiento 1) y 18 (tratamiento 2) observaciones (experimento 2). Pococí, Limón, Costa Rica 2008-2009.

\begin{tabular}{lcccc}
\hline Tipo de funda & Firmeza $^{1}$ & \multicolumn{3}{c}{ Color $^{\mathbf{2}}$} \\
\cline { 3 - 5 } & & $\mathbf{L}^{*}$ & $\mathbf{a}^{*}$ & $\mathbf{b}^{*}$ \\
\hline Santa Lucía & 47,11 & 54,46 & $-14,33$ & 31,75 \\
Fotosensible & 47,56 & 56,48 & $-14,23$ & 33,23 \\
Error Estándar & 0,44 & 0,50 & 0,20 & 65 \\
Probabilidad & 0,6268 & 0,0109 & 0,7414 & 0,1011 \\
\hline
\end{tabular}

\footnotetext{
${ }^{1}$ Medido en ambos experimentos, interacción experimento $\mathrm{x}$ tratamiento: $\mathrm{P}=0,3746$ por lo que el conjunto de datos se presenta ignorando el factor experimento.

${ }^{2}$ Medido solo en el experimento 2, en donde la coordenada $\mathrm{L}^{*}$ es una medida de la claridad (blanco-negro y varía desde el punto de ausencia de reflejo, $\mathrm{L}^{*}=0$, hasta el punto de reflejo difuso perfecto, $\mathrm{L}^{*}=100$ ). La escala $\mathrm{a}^{*}$ varía desde los valores negativos para el color verde, hasta los positivos para el rojo. La escala $b^{*}$ varía desde los valores negativos para el color azul, hasta los positivos para el amarillo.
}

(2006) quienes encontraron con la funda azul frutos de mayor peso en comparación con la transparente y del señalado por Chillet y Jannoyer (1996) quienes determinaron también con la funda azul frutos de mayor peso y dimensiones con respecto a la transparente fotoselectiva. Es importante añadir que tanto en el presente trabajo como en el realizado por Chillet y Jannoyer (1996) las fundas transparentes fotoselectivas fueron de similares características (polietileno de 20,3 $\mu$ de grosor y con aditivos para reducir luz UV e IR). 
La apariencia del racimo, a diferencia de la mayor incidencia de daño por sol mencionado por Weerasinghe y Ruwanpathirana (2002) en fundas sin color (transparentes), no difirió en este estudio entre las fundas evaluadas (azul y transparente fotoselectiva). Esta condición estaría explicada en función del mayor grosor del polietileno de la funda transparente fotoselectiva que no permitió la formación de depósitos de agua sobre el racimo y a los aditivos reductores de luz ultravioleta e infrarroja incorporados en ella. También es probable que este resultado esté parcialmente influenciado por la suficiente cantidad de hojas presentes en la planta durante el transcurso del desarrollo del racimo, situación que incidió en que la protección del racimo embolsado a la radiación solar fuera también mayor.

De acuerdo con Johns (1996), en algunas zonas de Australia los productores acostumbran cubrir simultáneamente con dos fundas al racimo en el invierno con el propósito de mitigar los efectos del frío. Estudios realizados por dicho autor, señalan que dicha práctica no afectó el peso del fruto con respecto a aquella en donde se colocó solo una funda al racimo. Este resultado podría considerarse también en función de esta investigación, donde en uno de los experimentos, el desarrollo del racimo de banano se efectuó en una época climática de temperaturas muy bajas $\left(29^{\circ} \mathrm{C}\right.$ y $17^{\circ} \mathrm{C}$, promedio de temperaturas máxima y mínima, respectivamente) para las condiciones climáticas propias del trópico húmedo que ocurren en la zona, sin determinarse, aún bajo esas condiciones tan adversas, diferencias productivas entre la funda de menor grosor (azul) con la de mayor grosor (transparente fotosensible) del polietileno.

Esta ausencia de efecto de los diferentes grosores de polietileno sobre las variables productivas coincide con lo señalado por Soto (1992) quien menciona que su selección estaría más en función del costo del material o de una alta luminosidad. Al respecto González $(2010)^{3}$, bajo condiciones climáticas del Pacífico Seco de Costa Rica (temperatura y luminosidad incidente máximas de $34,6^{\circ} \mathrm{C}$ y 72,6 lux, respectivamente), debió sustituir, debido a la destrucción del polietileno por efecto de la radiación solar, las fundas convencionales azules con un grosor de polietileno de 12,7 $\mu$ por

\footnotetext{
3 González, M. 2010. Proyecto de banano en la provincia de Guanacaste. Pococí, Limón, Costa Rica, Corporación Bananera Nacional. Comunicación personal.
}

fundas de mayor grosor $(17,8 \mu)$ y con aditivos para reducir el efecto de la luz ultravioleta e infrarroja.

Los resultados de este estudio coinciden con los de Vargas et al. (2010) quienes indican que las dimensiones, la presentación y la calidad poscosecha de los frutos de banano (Musa AAA, cvs. Grande Naine y Williams) no fueron afectados por el color ni por la densidad del polietileno de las fundas en estudios realizados por la Corporación Bananera Nacional de Costa Rica, y con aquellos descritos por Weerasinghe y Ruwanpathirana (2002) quienes señalan que el color y tipo del material no afectó el intervalo de días de embolse a cosecha, el peso del racimo o de sus frutos ni los grados brix de banano (Musa AAB, cv Mysore). Sin embargo, difieren en la respuesta productiva antes mencionada de aquellos estudios publicados por Soto (1992), Chillet y Jannoyer (1996), Daza y Cayón (2004), Daza y Cayón (2006) en bananos (Musa AAA) del subgrupo Cavendish ya señalados.

La diferencia entre resultados experimentales podría estar dada con base a la metodología experimental usada. En el presente estudio los tratamientos no se designaron como parte de una parcela experimental compuesta por un número determinado de plantas y por el contrario se aplicaron en grupos de racimos con una misma edad de embolse y de manos presentes luego del desmane (racimos equivalentes), asignando dichos tratamientos de manera rotativa y aleatoria conforme la aparición del tamaño de racimo requerido. También se consideró el mayor número de repeticiones posible, cada una de las cuales estuvo constituida por un racimo. Además el conjunto de datos se analizó considerando la posición de la mano en el racimo (manos equivalente) sugerida por Vargas y Blanco (2000). Esto permitió eliminar las fuentes de invalidación dadas por el clima, el suelo, el vigor de la planta y el racimo así como fortalecer el análisis de los datos en función de una comparación entre racimos de un mismo tamaño.

En contraste, en experimentos de este tipo donde cada tratamiento está constituido por parcelas de plantas, la expresión de las fuentes de invalidación antes mencionadas puede tener una mayor influencia sobre los resultados. Además, bajo esta perspectiva generalmente la cantidad de repeticiones o parcelas es menor, situación que afecta la fortaleza del análisis de los datos, que ya de por sí, presentan una alta variación. 
De esa forma, y considerando las condiciones agroclimáticas del Caribe de Costa Rica así como la metodología de evaluación y análisis de los experimentos, no fue posible determinar en este estudio diferencias en el peso del racimo, en el grosor y longitud del fruto central de la fila externa de las manos segunda, cuarta y sexta, en la apariencia del fruto ni en su color entre fundas de polietileno de uso tradicional (Azul Santa Lucía) y transparentes fotoselectivas. Además, los resultados son claros en señalar, que un mayor grosor de polietileno no mitiga en el racimo de banano, los efectos de las bajas temperaturas como las que ocurren en el Caribe costarricense. Ante tal perspectiva se debe considerar también el costo del material, el cual aumenta en función de su grosor y, tal como se demostró en este estudio, no representó ninguna ventaja sobre otros tipos de fundas con polietileno de menor grosor y costo más bajo.

\section{AGRADECIMIENTOS}

Los autores agradecen a la gerencia y al personal de la Finca la Teresa, propiedad de Banacol S.A. por la colaboración prestada.

\section{LITERATURA CITADA}

Campbell, SJ; Williams, WT. 1976. Factors associated with maturity bronzing of banana fruit. Australian Journal of Experimental Agriculture and Animal Husbandry 16:428-32.

Cayón, G. 2007. Influencia de las bolsas de polietileno sobre el desarrollo y carbohidratos de los frutos de plátano. In Murillo, R; Bejarano, AL; Morales, H; Pubiano, J. eds. Memorias Congreso Mundial de Banano y Plátano. Montenegro, CO. p. 13.

Chillet, M; Jannoyer, M. 1996. Selección de bolsas para optimizar las condiciones de crecimiento del banano. InfoMusa 5(1):25-27.

Daniells, JW; O’Farrel, PJ; Mulder, JC; Campbell, SJ. 1987. Effect of bunch covering and bunch trimming on bananas in north Queensland. Queensland Journal of Agricultural and Animal Sciences 44(2):101-105.

Daniells, JW; Lisle, T; O'Farrel, PJ. 1992. Effect of bunch covering methods on maturity bronzing, yield and fruit quality of bananas in north Queensland. Australian Journal of Experimental Agriculture 32:121-5.
Daniells, JW; Lindsay, S. 2005. Banana bunch covering. (en línea). DPI note. Department of Primary Industries. Queensland, Australia. Consultado 21 enero 2010. Disponible http://www2.dpi.qld.gov.au/ horticulture/4988.html.

Daza, MA; Cayón, G. 2004. Influencia del color de las bolsas de polietileno sobre las características físicas y químicas de los frutos de plátano y banano en época de verano e invierno en tres localidades (norte-centrosur) de la región de Urabá. Universidad Nacional de Colombia. Sede Bogotá. Facultad de Agronomía. Informe final. $72 \mathrm{p}$.

Daza, MA; Cayón, G. 2006. Efecto del color de las bolsas de polietileno sobre las características físicas y químicas de frutos de banano. In Soprano, E; Adami, F; Lichtemberg, L; Silva, M. eds. XVII Reunião Internacional da Associação nas Pesquisas sobre banana no Caribe e na América Tropical. ACORBAT, Santa Catalina, BR. p. 319.

Galán Sauco, V; Cabrera, J; Gómez, PM. 1996. The evaluation of different bunch covers (Musa acuminata) in the Canary Islands. Fruits 51(1):13-24.

Guevara, E; Rodríguez, W. 2006. Arquitectura vegetal e interceptación de luz. In Villalobos, E. ed. Fisiología de la producción de los cultivos tropicales. Vol. 6. Editorial de la Universidad de Costa Rica. San José, CR. 77 p.

InfoStat. 2002. Versión 1.1. Grupo Info-Stat, Facultad de Ciencias Agropecuarias, Universidad Nacional de Córdoba, Argentina. 216 p.

Jannoyer, M; Chillet, M. 1998. Amélioration de la croissance des bananes par utilization de la gaine Katryx ${ }^{\circledR}$. Fruits 53:219-228.

Johns, GG. 1996. Effects of bunch trimming and double bunch covering on yield bananas during Winter in New South Wales. Australian Journal of Experimental Agriculture 36:229-235.

Lara, F. 1970. Problemas y procedimientos bananeros en la zona Atlántica de Costa Rica. Imprenta Trejos. San José, CR. 278 p.

Ministry of Fisheries, Crops and Livestook. 2003. Bunch covers for improving plantain and banana peel quality. Postharvest Handling Technical Series. Technical Bulletin No. 4. Georgetown, Guyana. 9 p.

Robinson, JC. 1996. Bananas and plantains. CAB International, Wallingford, WK. 238 p.

SAS Institute Inc. 2002-2003. Version 9.1.3. SAS Institute Inc., Cary, NC, USA. 
Soto, M. 1992. Siembra y operaciones de cultivo. In Soto, M. ed. Bananos: cultivos y comercialización. 2 ed. Litografía e Imprenta LIL. San José, CR. 649 p.

Serrano, E; Segura, R; Ortega, R.; Sandoval, J. 2008. Modelo de restitución del potasio removido en la fruta fresca exportada en una plantación de banano de alta productividad. p. 106-110. In Sandoval, J. ed. Informe Anual 1997. Dirección de Investigaciones. Corporación Bananera Nacional (CORBANA, S.A.). San José, CR. 321 p.

Vargas, A; Blanco, F. 2000. Consideraciones metodológicas para la evaluación del desmane en banano (Musa AAA, $c v$ 'Valery). InfoMusa 9(2):19-21.

Vargas, A; Blanco, F. 2004. Metodologías para estimar la intensidad de desmane en racimos de banano (Musa AAA, $c v$ Valery). CORBANA 30(57):107-119.
Vargas, A; Valle, H; González, M. 2010. Efecto del color y de la densidad del polietileno de fundas para cubrir el racimo sobre dimensiones, presentación y calidad poscosecha de frutos de banano y plátano. Agronomía Costarricense 34(2):269-285.

Von Loesecke, H. 1950. Bananas. InterScience Publishers, New York, USA. 189 p.

Wade, N; Kavanagh, E; Tan S. 1993. Sunscald and ultraviolet light injury of banana fruits. J. Hort. Sci. 68 (3): 409-419.

Weerasinghe, SS; Ruwanpathirana, KW. 2002. Influence of bagging material on bunch development Banana (Musa spp.) under high density planting system. Annals of Sri Lanka Department of Agriculture 4: 47-53. 
